\title{
Acute Suppurative Sialadenitis Secondary to Excisional Ranula Biopsy in Child
}

\author{
${ }^{1}$ Tiago N Pinheiro, ${ }^{2}$ Juliana Arid, ${ }^{3}$ Aloizio P Maciel, ${ }^{4}$ André FM Machado, ${ }^{5}$ Marleno L Monteiro \\ ${ }^{6}$ Mariana de Oliveira Daltoé, ${ }^{7}$ Fabricio K de Carvalho
}

\begin{abstract}
Introduction: Ranulas are lesions that affect the salivary glands, and they are generally treated by excision; however, they can recur in some cases where the gland is not removed and they may progress to acute suppurative sialadenitis, yet such cases are rare, especially in children.
\end{abstract}

Aims: To report clinical management of occurrence of acute suppurative sialadenitis secondary to excisional biopsy of ranula in a child.

Case report: An 11-year-old female patient was diagnosed with ranula where excisional biopsy was performed. Subsequently, the patient developed infection, suggesting Ludwig's angina, after reevaluation, she was diagnosed with acute suppurative sialadenitis in the right mandibular gland; during the follow-up period, antibiotic therapy was introduced.

Conclusion: The close relationship of the biopsy site with the submandibular space and other structures of the neck required the completion of antibacterial prophylaxis associated with preoperative care, transoperative care, and postoperative care to combat opportunistic infections and their subsequent complications.

Clinical significance: Suppurative sialadenitis and its subsequent complications may be considered, although rare, as a complication in cases of ranula.

Keywords: Ludwig's angina, Ranula, Sialadenitis.

How to cite this article: Pinheiro TN, Arid J, Maciel AP, Machado AFM, Monteiro ML, de Oliveira Daltoé M, de CarvalhoFK. Acute Suppurative Sialadenitis Secondary to Excisional Ranula Biopsy in Child. Int J Experiment Dent Sci 2016;5(2):123-126.

Source of support: Nil

Conflict of interest: None

\footnotetext{
${ }^{1,4,7}$ Professor, ${ }^{2,6}$ Postgraduate Student, ${ }^{3}$ Graduate Student ${ }^{5}$ Private Practioner

1,6,7 Department of Pediatric Dentistry, University of São Paulo School of Dentistry of Ribeirão Preto, Ribeirão Preto, São Paulo, Brazil

2,3 School of Dentistry, University of the State of Amazonas Manaus, Amazonas, Brazil

${ }^{4}$ School of Dentistry, Universidade Nilton Lins, Manaus, Amazonas Brazil

${ }^{5}$ School of Dentistry, Hospital João Lúcio, Manaus, Amazonas Brazil

Corresponding Author: Juliana Arid, Postgraduate Student School of Dentistry, University of the State of Amazonas Manaus, Amazonas, Brazil, Phone: +551636023995 , e-mail: juliana_arid@hotmail.com
}

\section{INTRODUCTION}

Ranulas are lesions that affect the salivary glands and their ducts located below the mouth floor. In general, retention and mucus extravasation are responsible for the origin of the lesion in these structures, and there may be an association between the appearance of ranulas with previous surgery or trauma. The term "ranula" is given because of its blue tint, which refers to the abdomen of a frog. ${ }^{1,2}$

More often oral ranula affects more the sublingual gland, with an oval swelling of purplish color, located in the mouth floor, floating palpation, resembling the back of a frog. ${ }^{1,2}$ Ranulas usually have small dimensions, not exceeding $3 \mathrm{~cm}$ in most cases. This type of lesion occurs especially between 10 and 19 years of age, affecting mainly females. ${ }^{1,2}$

The most common treatment options for ranula are marsupialization, excision of the lesion, excision of sublingual gland, or a combination of the aforementioned excisions. Treatments that do not involve removal of sublingual gland result in increased recurrence, as this type of excision cures all ranulas. However, many surgeons opt for a more conservative treatment due to potential surgical complications that can occur after the removal of sublingual glands. These complications include injury to the lingual nerve and injury to the Wharton duct with the possibility of stenosis leading to sialadenitis. ${ }^{3}$

The anatomical relationship of Wharton's duct and the submandibular gland itself with the submandibular space provides, in cases of infection, rapid infectious dissemination to other adjacent structures. Therefore, an immediate process of intervention is necessary to prevent future complications. ${ }^{3,4}$

Sialadenitis is defined as inflammation of salivary glands, and their most common causes are viral parotitis and juvenile recurrent parotitis. However, there are other causes that should be taken into account, such as bacterial infections, sialolithiasis, and traumatic ranulas, this being the cause in fewer cases. Bacterial sialadenitis occurs mostly in the parathyroid gland and is characterized by marked swelling that extends from the cheek to the angle of the jaw. ${ }^{5,6}$

Sialadenitis can result in edema in the mouth floor possibly impairing breathing and can be distinguished from other inflammatory diseases affecting the salivary glands due to the presence of pus. In the absence of purulence, fever, and leukocytosis, the diagnosis must be maintained as sialadenitis. ${ }^{5}$ 
Sialadenitis in children is very rare, affecting less than $5 \%$ of cases; as the similarity of sialadenitis stones in adults are found in the submandibular glands in 89 to $90 \%$ of cases, followed by glandular parathyroid, and rarely in the sublingual gland and in the minor salivary glands. ${ }^{5}$ The incidence of acute suppurative sialadenitis is not very clear, but it is extremely rare, as it comprises $0.01 \%$ of all cases treated in hospitals. Although, it has no gender preference, color, and race, it occurs more commonly in older, weak, and dehydrated patients. ${ }^{7}$

The most common signs and symptoms of acute sialadenitis are swelling, pain, fever, and erythema overlying the affected glands. They are usually unilateral, and in cases where the signs and symptoms are bilaterally present, they are most prevalent on one side. ${ }^{5}$

This study aims to report the complications that occurred after excisional biopsy of a ranula and the proposed treatment at each stage of this case.

\section{CASE REPORT}

An 11-year-old female patient was complaining of swelling under the tongue. During intraoral examination, a bubble of approximately $1.5 \mathrm{~mm}$ in diameter in the right sublingual region was detected, a normochromic sessile base, a clear and continuous edge with a smooth, shiny texture (Fig. 1A). The initial clinical diagnosis suggested the presence of a ranula. After verifying the systemic normality of the patient through laboratory tests, the proposed treatment was excisional biopsy. After obtaining parental consent, the procedure was performed and the patient received oral hygiene instructions (Figs 1B and 1C).

After excisional biopsy of the area, the histopathological examination was performed confirming the clinical diagnosis of ranula, since the histopathological report was consistent with a mucous retention cyst (Fig. 1D).

Proper postoperative care was passed to the patient along with the prescription of $500 \mathrm{mg}$ of sodium dipyrone and $100 \mathrm{mg}$ of nimesulide.

Three days after the procedure, the patient returned reporting the presence of swelling, trismus, erythema, and progressive pain after surgery together with systemic manifestations of infection. The patient was requested to return for a reevaluation, but did not return.
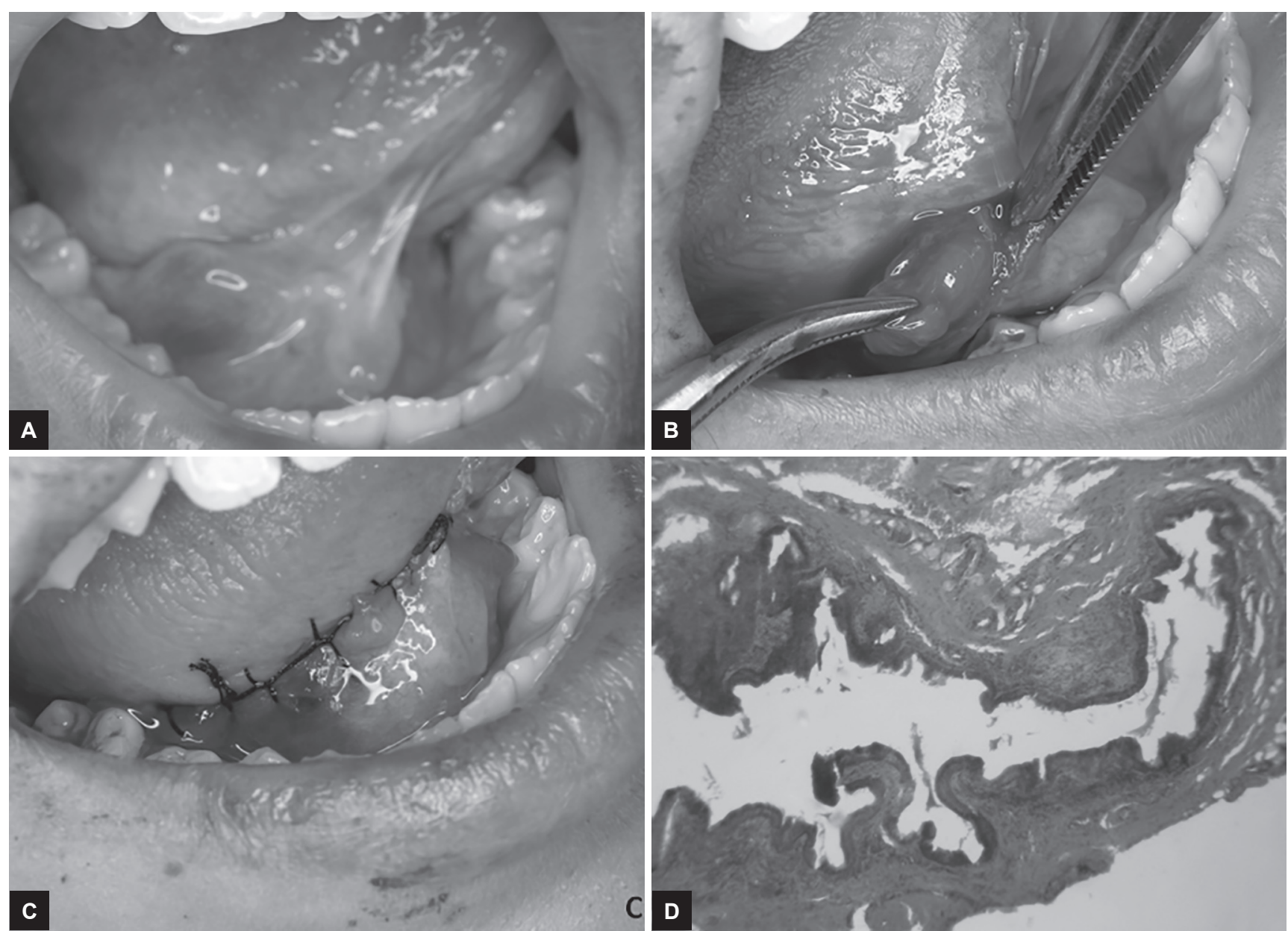

Figs 1A to D: (A) Ranula in the right sublingual gland region; (B) surgical excision of the lesion; (C) suture; and (D) microscopic examination of the lesion, thus confirming the diagnosis (hemotoxylin and eosin stained; 40x) 

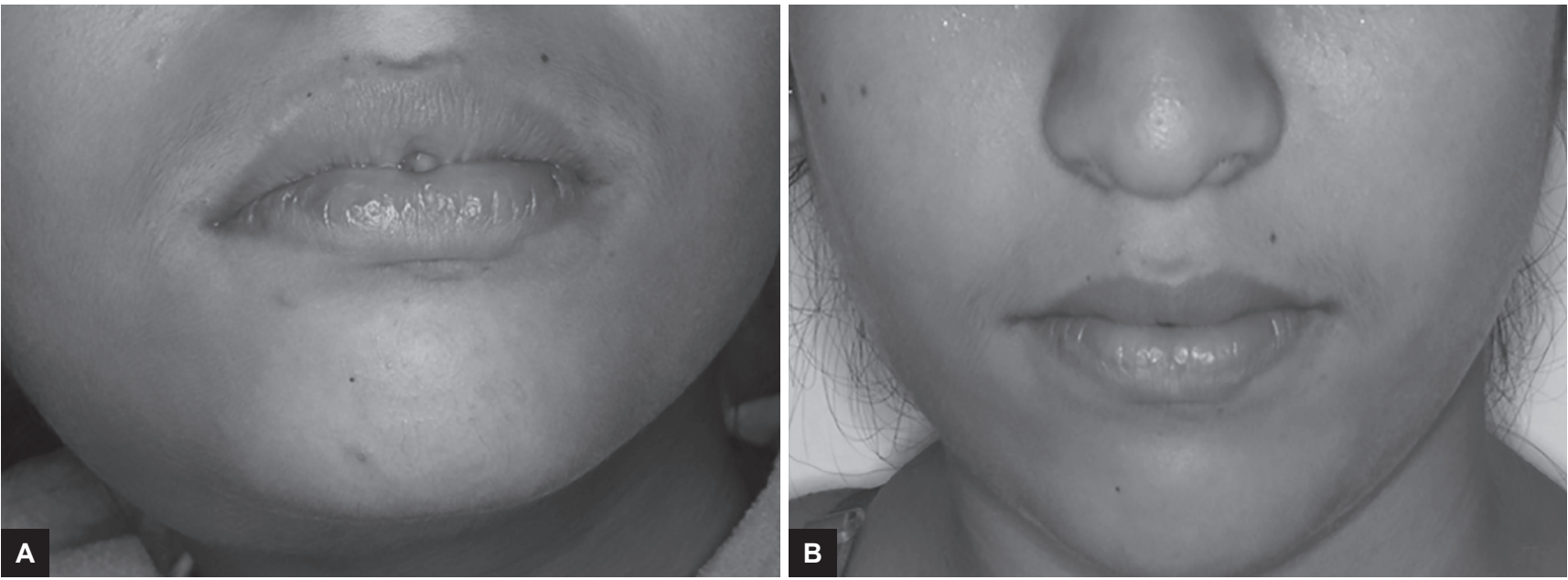

Figs 2A and B: (A) Facial asymmetry, swelling in the right cervical region of the patient showing clinical diagnosis of acute suppurative sialadenitis; (B) five months of follow-up without recurrence

Six days after the surgery, the patient arrived at the emergency department and was hospitalized on suspicion of Ludwig's angina. On admission, the patient was treated with third-generation ceftriaxone intravenous $1.250 \mathrm{mg}$ every 12 hours administered for 7 days.

After being discharged from the hospital on the 7th day, the patient was evaluated. The evaluation revealed the presence of facial asymmetry, swelling of the right cervical region (Fig. 2A) associated with trismus, an 18-mm mouth opening, cervical ganglia infarction, difficulty in moving the neck, and pain. Acute suppurative sialadenitis was diagnosed in the same submandibular gland that was previously affected by the ranula. The patient reported having difficulties and fear of performing good oral hygiene after surgery. The proposed thermotherapy treatment was associated with $500 \mathrm{mg}$ of amoxicillin and $125 \mathrm{mg}$ of potassium clavulanate.

Due to the treatment given, there was decrease in trismus and other clinical signs were observed in the following week. However, the involved submandibular gland showed up floating and rigid palpation with reduced salivary flow. The follow-up after 5 months showed a reduction of glandular volume, but salivary flow showed no significant changes (Fig. 2B).

\section{DISCUSSION}

Among the existing surgical modalities for ranula, there are greater numbers of relapse when treatment option consists of excision or marsupialization of the lesion. ${ }^{1-3}$ However, there are cases where such procedures may be indicated in order to preserve the adjacent anatomical structures as well as the functioning of the gland. ${ }^{1-3}$ The excision of the submandibular gland with or without removal of the ranula has shown more lasting results. ${ }^{1-3}$ In this case, only the action of excision of the ranula without association with submandibular gland was determined, thereby aiming to preserve salivary structures and obtain a correct diagnosis of the case.

The difficulty of cleaning and the fear of rupturing points in the brushing action contributed in this case to the surgical site infection, which developed into acute suppurative sialadenitis. Another possible contributing factor was the lack of commitment of responsibility in returning for a review of the manifested complications. The immediate intervention of the infection could have avoided major complications. ${ }^{3}$ Although it is more common in older and immune compromised people, this did not happen in our case as the patient was only 11 years old. This case demonstrates a rare occurrence of acute suppurative sialadenitis in children; this occurs mainly due to the evolution of sialolithiasis. ${ }^{6-9}$

The etiology of acute suppurative sialadenitis is varied, and it may result from decreases in salivary flow caused by, e.g., a small dehydration leading to inflammation and tissue destruction ${ }^{5,7,9}$; viral and bacterial infections can cause infections leading to inflammation of the salivary gland, as occurred in the case described here. 5,9,10 Other causes are suggested as genetic factors, immune disorders, and congenital anomalies of the duct. ${ }^{5}$ The submandibular infections can occur due to dental infections, lymphadenitis, trauma, and subsequent surgeries as shown in the case. ${ }^{4}$

In this case, it is shown that the acute suppurative sialadenitis ranula occurred after excision, and after this procedure, due care was not taken, thereby providing a bacterial colonization. The duct obstruction by a sialolith, or even surgical damage of Wharton's duct, also provides better chances for installation and appearance of bacterial infection in the salivary glands. After the involvement of this anatomical region, which is the submandibular gland and Wharton's duct, the infection has a rapid dissemination to other structures of the neck, 
where the fascias that encounter the muscles present in that area end up forming infectious dissemination routes. ${ }^{3,5,9}$ Other articles mention the appearance of acute suppurative sialadenitis as a possible complication of an unsuccessful ranula treatment; however, to the best of our knowledge, there is no study reporting the situation.

Ludwig's angina may be the consequence of the spread of infection to the submandibular gland, which is characterized by diffused aggressive cellulite affecting the submental, submandibular, and sublingual spaces bilaterally together with the presence of edema, erythema, and systemic signs of infection and trismus. ${ }^{4}$

On admission, the patient was diagnosed with suspected Ludwig's angina; therefore, rapid engagement was performed by third-generation intravenous ceftriaxone in an attempt to contain the infection, since the collapse of airways is often reported in cases where there is an evolving infection. It is mandatory to take precautions in cases of infection of the submandibular space to prevent the development and complications of the infection is warranted. ${ }^{4}$ The severity of the case was due to the excision of the ranula, where after the surgical procedure, due care was not taken and the patient developed an infection framework, thus leading to Ludwig's angina.

The culture of bacteria in cases of opportunistic infections of the submandibular gland and submandibular space has most frequently isolated Staphylococcus aureus as the primary infectious agent. This group of bacteria, as well as other types present in this type of infection, is a producer of $\beta$-lactamase, which inhibits the action of antibiotics from the group of penicillin and cephalosporins. ${ }^{4,5,9}$ For this reason, amoxicillin and potassium clavulanate have been used in the treatment of patients in this study, as ceftriaxone could not help any infection. Pharmacological treatment was associated with thermotherapy in an attempt to stimulate the salivary flow and assist in draining the infection.

\section{CONCLUSION}

Dentistry is varying with induction of modern science to practice dentistry. ${ }^{10,11}$ Although ranula biopsies are fast procedures with low complexity, close relationship of the mouth floor with the submandibular space and other neck structures associated with the low socioeconomic condition of the patient necessitates the implementation of antibacterial prophylaxis associated with preoperative, transoperative, and postoperative care to combat opportunistic infections.

\section{CLINICAL SIGNIFCANCE}

The suppurative sialadenitis and its subsequent complications may be considered, although rare, as a complication in cases of ranula.

\section{REFERENCES}

1. Zhao YF, Jia Y, Chen XM, Zhang WF. Clinical review of 580 ranulas. Oral Surg Oral Med Oral Pathol Oral Radiol Endod 2004 Sep;98(3):281-287.

2. Jia Y, Zhao Y, Chen X. Clinical and histopathological review of 229 cases of ranula. J Huazhong Univ Sci Technolog Med Sci 2011 Oct;31(5):717-720.

3. Zhao YF, Jia J, Jia Y. Complications associated with surgical management of ranulas. J Oral Maxillofac Surg 2005 Jan;63(1):51-54.

4. Boscolo-Rizzo P, Da Mosto MC. Submandibular space infection: a potentially lethal infection. Int J Infect Dis 2009 May;13(3):327-333.

5. Francis CL, Larsen CG. Pediatric sialadenitis. Otolaryngol Clin North Am 2014 Oct;47(5):763-778.

6. Laskawi R, Schaffranietz F, Arglebe C, Ellies M. Inflammatory diseases of the salivary glands in infants and adolescents. Int J Pediatr Otorhinolaryngol (Ireland) 2006 Jan;70(1): 129-136.

7. Chandak R, Degwekar S, Chandak M, Rawlani S. Acute submandibular sialadenitis - a case report. Case Rep Dent 2012;2012:615375.

8. Kaban LB, Mulliken JB, Murray JE. Sialadenitis in childhood. Am J Surg 1978 Apr;135(4):570-576.

9. Troeltzsch M, Pache C, Probst FA, Ehrenfeld M, Otto S. Antibiotic concentrations in saliva: a systematic review of the literature, with clinical implications for the treatment of sialadenitis. J Oral Maxillofac Surg 2014 Jan;72(1):67-75.

10. Brook I. Aerobic and anaerobic microbiology of suppurative sialadenitis. J Med Microbiol 2002 Jun;51(6):526-529.

11. Saini R. Ozone therapy in dentistry: a strategic review. J Nat Sc Biol Med 2011; 2:151-153. 